\title{
Promoter Specificity and Efficacy in Conditional and Inducible Transgenic Targeting of Lung Macrophages
}

\author{
Alexandra L. McCubbrey ${ }^{1,2 *}$, Kristen C. Allison ${ }^{3}$, Alisa B. Lee-Sherick ${ }^{3}$, \\ Claudia V. Jakubzick ${ }^{4}$ and William J. Janssen ${ }^{1,2}$ \\ ${ }^{1}$ Department of Medicine, National Jewish Health, Denver, CO, United States, ${ }^{2}$ Division of Critical Care Medicine and \\ Pulmonary Sciences, University of Colorado Denver, Denver, CO, United States, ${ }^{3}$ Department of Pediatrics, University \\ of Colorado Anschutz Medical Campus, Aurora, CO, United States, ${ }^{4}$ Department of Pediatrics, National Jewish Health, \\ Denver, CO, United States
}

OPEN ACCESS

Edited by: John William Christman, The Ohio State University Columbus, United States

Reviewed by:

Myung-Hee Kwon, Ajou University, South Korea Arnaud Millet, INSERM UMR5309 Institut pour l'Avancée des Biosciences $(I A B)$, France

${ }^{*}$ Correspondence: Alexandra L. McCubbrey mccubbreya@nihealth.org

Specialty section:

This article was submitted to Inflammation,

a section of the journal

Frontiers in Immunology

Received: 03 October 2017 Accepted: 08 November 2017 Published: 24 November 2017

Citation:

McCubbrey AL, Allison KC, Lee-Sherick $A B$, Jakubzick CV and

Janssen WJ (2017) Promoter Specificity and Efficacy in

Conditional and Inducible

Transgenic Targeting of Lung Macrophages.

Front. Immunol. 8:1618 doi: 10.3389/fimmu.2017.01618
Conditional and inducible Cre-loxP systems are used to target gene deletion to specific cell lineages and tissues through promoter-restricted expression of the bacterial DNA recombinase, Cre. Although Cre-loxP systems are widely used to target gene deletion in lung macrophages, limited data are published on the specificity and efficiency of "macrophage targeting" Cre lines. Using R26-stop ${ }^{\mathrm{f} / \mathrm{l} / \mathrm{I}}$-TdTomato and tetOn-GFP reporter lines, we assessed the specificity and efficiency of four commercially available Cre driver lines that are often considered "macrophage specific." We evaluated two conditional (Csf1r-Cre and LysM-Cre) and two inducible [CX ${ }_{3} \mathrm{CR} 1$-estrogen receptor-Cre (ERCre) and CD68-rtTA] lines. We assessed Cre activation in six resident lung myeloid populations, as well as activation in lung leukocytes, lung epithelial and endothelial cells, peripheral blood leukocytes, and tissue macrophages of the spleen, bone marrow, and peritoneal cavity. Although Csf1r-Cre and LysM-Cre target resident alveolar macrophages (ResAM) and interstitial macrophages (IM) with high efficiency, neither line is specific for macrophages. Csf1r-Cre targets all leukocyte populations, while LysM-Cre targets dendritic cell, neutrophils, monocytes, and a quarter of lung epithelial cells. $\mathrm{CX}_{3} \mathrm{CR} 1$-ERCre and CD68-rtTA both target IM, but do not target ResAM. Further, although neither line is specific for macrophages, a pulse-wait administration of tamoxifen or doxycycline can be used to significantly improve IM specificity in these inducible lines. In summary, while Cre-loxP remains a powerful tool to study macrophage function, numerous pitfalls exist. Herein, we document strengths and weaknesses of Csf1r-Cre, LysM-Cre, $\mathrm{CX}_{3} \mathrm{CR} 1-\mathrm{ERCre}$, and CD68-rtTA systems for targeting specific macrophage populations in the lungs and provide data that will aid investigators in selecting the proper strain.

Keywords: macrophage, Cre, lung, conditional, LysM-Cre, CX3CR1-estrogen receptor-Cre, CD68-rtTA, Csf1r-Cre

\section{INTRODUCTION}

In vivo studies are critical for understanding the homeostatic functions of lung macrophages and their roles in pulmonary disease. In this context, conditional transgenic targeting of cell populations using a Cre-loxP strategy is one of the most powerful tools available for in vivo studies of macrophages. However, recent work has exposed the limited macrophage specificity of many 
"macrophage targeting" Cre driver lines (1-3). Accurate data interpretation requires a firm understanding of the underlying genetic principles of Cre-loxP mice and detailed knowledge regarding the limitations of the chosen Cre driver.

Cre is a bacterial recombinase that catalyzes recombination between paired DNA recognition sites termed LoxP sites $(4,5)$. Restricted Cre expression can be achieved through the use of a cell-specific promoter that drives expression of Cre only in the cells that activate the chosen promoter; this is termed a conditional transgene. When a mouse line with a conditional Cre driver is crossed with a line in which a gene of interest is "floxed" with LoxP sites, the floxed gene is deleted only in cells where Cre is expressed. As this change is genomic, it will remain through the life of the targeted cell and all daughter cells.

Conditional inducible systems represent an enhancement over standard conditional systems by enabling temporal control of transgenes. The two most common include estrogen receptorCre (ERCre) (6) and tetracycline-On-Cre (tet-On-Cre) $(7,8)$. Conditionally expressed ERCre requires tamoxifen as a cofactor for Cre activity; until tamoxifen is administered, the mouse remains, essentially, "wild-type." Similarly, tet-On-Cre systems require the administration of doxycycline to allow a conditionally expressed rtTA to bind to a tet-On promoter and induce Cre expression. However, conditional and conditional inducible systems are only as strong as the chosen conditional promoter. It has become increasingly clear that macrophage "specific" Cre driver lines have promiscuous activation in a broad range of myeloid cells $(1-3,9)$.

During homeostasis, the lung contains at least six unique extravascular myeloid cell populations that are defined by their location and site of origin, and distinguished by their cell surface marker repertoires. Resident alveolar macrophages (ResAM) reside in the airspace lumen and arise during embryogenesis from fetal liver monocytes (10-12). In comparison, three subpopulations of interstitial macrophages (IM) are located in the lung tissue and are believed to replenish more readily, throughout the lifespan of the mouse, from circulating Ly6C $\mathrm{C}^{\text {hi }}$ monocytes $(13,14)$. Notably both ResAM and IM are capable of self-renewal and are maintained with limited contribution from adult bone marrow-derived monocytes $(11,14)$. IM can be divided into subpopulations based on their expression of CD11c, CD206, and MHCII: IM1 (CD206 $\left.{ }^{+} \mathrm{MHCII}^{-}\right)$,

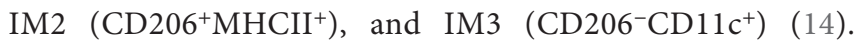
Dendritic cells (DCs) comprise the remaining myeloid cells in the homeostatic lung and include CD $11 \mathrm{~b}^{+}$and $\mathrm{CD} 103^{+} \mathrm{DC}$ subsets $(15,16)$.

In the setting of inflammation, additional myeloid cells infiltrate the lung tissues, including neutrophils, eosinophils, and monocytes. The absolute number of macrophages in the lung tissue also expands (17), presumably driven by infiltrating monocytes. Further, a second macrophage population appears in the alveolus, termed recruited macrophages (RecM $\Phi$ ) $(18,19)$. Prior research suggests that these RecM $\Phi$ differentiate from infiltrating Ly6 $\mathrm{C}^{\text {hi }}$ monocytes (20-22). Clear surface markers have been established to separate ResAM, IM1, IM2, IM3, and RecM $\Phi$ from other myeloid cells $(14,23,24)$, but functional studies of macrophages in vivo remain a challenge.
To determine the optimum systems for targeting specific macrophage cell populations in the lungs, we selected four commercially available "macrophage" Cre driver lines. These included two conditional lines (Csf1r-Cre and LysM-Cre) and two inducible ones $\left(\mathrm{CX}_{3} \mathrm{CR} 1\right.$-ERCre and CD68-rtTA). While these "macrophage" targeting lines have been heavily used, the pitfalls of their use have been largely ignored, in part because a systemic analysis of their specificity in the lung has not been performed. We crossed these Cre driver lines with various fluorescent reporter lines, discussed in detail below, to assess Cre activity in lung macrophages during homeostasis and inflammation. We also examined peripheral blood, since current evidence suggests that circulating monocytes give rise to macrophage subsets during inflammation. Both LysM-Cre and Csf1r-Cre strongly targeted ResAM and IM. However, we found that Csf1r-Cre (25) non-specifically targeted all leukocytes, including lymphocytes, while LysM-Cre (26) problematically targeted neutrophils and a quarter of lung epithelial cells. Activation of $\mathrm{CX}_{3} \mathrm{CR} 1$-ERCre (11) and CD68-rtTA (27) was strongly observed in IM, but absent in ResAM. Therefore, while $\mathrm{CX}_{3} \mathrm{CR} 1$-ERCre and CD68-rtTA are promising models for the study of IM, all four systems struggle to specifically target Cre activity to ResAM.

\section{METHODS}

\section{Animals}

This study was approved and performed in accordance with the ethical guidelines of the Institutional Animal Care and Use Committee at National Jewish Health (approval \#AS2574-01-20). All mice were obtained from Jackson Laboratories (Bar Harbor, ME, USA) and bred at National Jewish Health. LysM-Cre (B6.129P2-Lyz2tm1(cre)Ifo/J; stock number 004781), Csf1rcre (FVB- $\mathrm{Tg}(\mathrm{Csf} 1 \mathrm{r}-\mathrm{icre}) 1 \mathrm{Jwp} / \mathrm{J}$; stock number 021024), and $\mathrm{CX}_{3}$ CR1-ERT2 (B6.129P2(C)-CX $\mathrm{C}_{3}$ CR1tm2.1(cre/ERT2)Jung/J; stock number 020940) mice were crossed with R26-stop pt/f $_{-}$ TdTomato(B6.Cg-Gt(ROSA)26Sortm14(CAG-tdTomato)Hze/J; stock number 007914) mice. Experimental animals from these three crosses were hemizygous for both genes. hCD68-rtTA (B6. Cg- $\mathrm{Tg}\left(\mathrm{CD} 68-\mathrm{rtTA2S}{ }^{*} \mathrm{M} 2\right) 3 \mathrm{Mpil} / \mathrm{Mmjax}$; stock number 32044JAX) and R26-M2rtTA (B6.Cg-Gt(ROSA)26Sortm1(rtTA $\left.{ }^{*} M 2\right)$ Jae/J; stock number 006965) mice were crossed with tetOnGFP (Tg(tetO-HIST1H2BJ/GFP)47Efu/J; stock number 005104) mice. Experimental animals from hCD68-rtTA x tetOn-GFP were homozygous for both genes, while R26-rtTA x tetOnGFP were hemizygous for both genes. Both male and female mice were used in these experiments.

\section{Doxycycline and Tamoxifen Administration}

Normal diet chow was replaced with doxycycline chow (Teklad laboratory diets, NJ, USA) for 1 week, then mice were analyzed on day 7 (pulse). Doxycycline chow was dosed at $625 \mathrm{mg}$ doxycycline/kg chow, designed to provide 2-3 mg of doxycycline/day based on consumption of 4-5 g chow/day. Additional analyses were performed after mice were returned to normal diet for 1 , 2, or 4 weeks (wait). Mice were allowed to eat chow ad libitum. 
Tamoxifen (Sigma) suspended in neutral oil was given at $0.25 \mathrm{mg} / \mathrm{g}$ of body weight by intraperitoneal (IP) injection. Mice were injected on day 1 , again on day 4 , then analyzed on day 7 (pulse), Additional analyses were performed 1, 2, or 4 weeks after the last tamoxifen injection (wait).

\section{Tissue Harvest}

Mice were euthanized by $\mathrm{CO}_{2}$ inhalation or IP injection of Fatal Plus (Vortech Pharmaceuticals, Dearborn, MI, USA). Peritoneal lavage (PL), bronchoalveolar lavage (BAL), lung, blood, bone marrow, and spleen were collected on ice in the following order. PL was collected in $10 \mathrm{~mL}$ of PBS containing $0.5 \mathrm{mM}$ EDTA, pelleted, and resuspended in HBSS containing $0.3 \%$ BSA and $0.3 \mathrm{mM}$ EDTA. BAL was collected in $5 \mathrm{~mL}$ of PBS containing $0.5 \mathrm{mM}$ EDTA, pelleted, and resuspended in HBSS containing $0.3 \%$ BSA and $0.3 \mathrm{mM}$ EDTA. Blood was collected by cardiac puncture using a syringe coated with $50 \mu \mathrm{L}$ of $100 \mathrm{mM}$ EDTA, then placed directly into $10 \mathrm{~mL}$ of PharmLyse buffer (BD Biosciences, San Jose, CA, USA) for $20 \mathrm{~min}$ on ice. After 20 min, blood cells were washed and resuspended in HBSS containing $0.3 \% \mathrm{BSA}$ and $0.3 \mathrm{mM}$ EDTA. Lungs were perfused by injecting $10 \mathrm{~mL}$ PBS through the right ventricle, after which lung tissue was visibly blanched. Lung tissue was finely chopped with a razor blade then incubated at $37^{\circ} \mathrm{C}$ in $1 \mathrm{~mL}$ of $0.4 \mathrm{mg} / \mathrm{mL}$ Liberase TM (Roche, Indianapolis, IN, USA) in RPMI for $25 \mathrm{~min}$. Following incubation, tissue was pipetted rapidly up and down to further disaggregate, then filtered through $100 \mu \mathrm{m}$ filters. Lung cells were pelleted and residual RBC were lysed for $30 \mathrm{~s}$ using $1 \mathrm{~mL}$ of BD PharmLyse (BD Biosciences, San Jose, CA, USA). BAL, lung, blood, and PL cells were washed in HBSS and resuspended in HBSS containing 0.3\% BSA and $0.3 \mathrm{mM}$ EDTA. Spleen tissue was chopped and mechanically dissociated, then filtered through $100 \mu \mathrm{m}$ filters. Bone marrow was flushed from the cavities of the femur and tibia with cold PBS and mechanically dissociated, then filtered through $100 \mu \mathrm{m}$ filters. Residual RBC in spleen and bone marrow were lysed for 10 min using $1 \mathrm{~mL}$ of Gey's Solution (0.155 M NH4Cl $10 \mathrm{mM}$ KHCO3 aqueous). The hemolysis wash was repeated. Spleen and $\mathrm{BM}$ cells were washed and resuspended in PBS containing $2 \%$ FBS, 2 mM EDTA, and 0.09\% $\mathrm{NaN}_{3}$.

\section{Flow Cytometry}

Flow cytometry was performed on single cell suspensions. Cells were protected from light and incubations were performed on ice. Single cell suspensions were treated with unlabeled CD16/CD32 for 30 min to block non-specific FcyR-mediated binding. Cells were stained with surface antibody panels to identify myeloid populations for $1 \mathrm{~h}$, and then washed. When necessary, cells were incubated with streptavidin-conjugated secondary antibody for another $45 \mathrm{~min}$, and then washed. HBSS containing $0.3 \%$ BSA and $0.3 \mathrm{mM}$ EDTA was used as a buffer for all incubations. Single cell suspensions of BAL, lung, blood, and PL were analyzed using an LSRII flow cytometer (BD) and FlowJo software (Tree Star, Ashland, OR, USA). Spleen and bone marrow cells were analyzed using a YETI cell analyzer (Propel Labs). IM1, IM2, IM3, and $\gamma \delta$ T cells were the smallest populations examined; data collection was performed such that $>400$ events for each of these populations were analyzed, for most populations $>5,000$ events were analyzed. Primary antibodies were used in various combinations as follows (source/clone): unlabeled CD16/32 (eBioscience/93), Ly6G (BD/IA8), MHCII (BD/114.15.2), F4/80 (ebioscience/BM8), CD45 (BD/30-F11), Ly6C (eBioscience/HK1.4), Siglec-F (BD/ E50-2440), CD11c (eBioscience/N418), CD11b (eBioscience/ M1/70), CD103 (eBioscience/2E7), CD115 (eBioscience/ AFS98), CD64 (Biolegend/X54-5/7.1), MerTK (R\&D/BAF591), CD3 (Biolegend/17A2), CD19 (eBioscience/eBio1D3), NK1.1 (Biolegend/PK136), $\gamma \delta T C R$ (eBioscience/eBioGL3), EpCAM (Biolegend/G8.8), CD31 (Biolegend/390), Thrombomodulin (R\&D/AF3894), CD206 (Biolegend/C068C2), Tim4 (Biolegend/ F31-5G3), and Gr-1 (BD/RB6-8C5). All primary antibodies were used at a 1:400 dilution except $\operatorname{EpCAM~}(1: 1,000)$, and MerTK (1:100). Streptavidin-conjugated FITC, AF647, and PE secondary antibodies (Jackson Immunolaboratories) were used at 1:200.

\section{LPS Injury}

LPS (Escherichia coli 055:B5; List Biological Laboratories, Campbell, CA, USA) was administered in a dose of $20 \mu \mathrm{g}$ in $50 \mu \mathrm{L}$ of PBS. LPS was instilled intratracheally (IT) using a modified gavage needle. Mice were sedated with isoflurane (Baxter, Deerfield, IL, USA) before IT instillations. Reporter expression was assessed in BAL, lung, and blood 6 days after LPS instillation.

\section{RESULTS}

\section{Conditional Cre Systems Target Lung Macrophages with Varying Specificity}

To improve our understanding of conditional and conditional inducible systems within the lung and aid in experimental design and interpretation, we assessed the specificity and strength of four different Cre driver lines for targeting macrophages. The two conditional lines selected, LysM-Cre and Csf1r-Cre, are the most highly published "macrophage targeting" lines in the literature $(3,9,28)$. The two inducible lines, $\mathrm{CX}_{3} \mathrm{CR} 1$-ERCre and CD68-rtTA, were developed more recently and have been used less extensively. However, based on previous work, we suspected that both lines might be appropriate for targeted studies of IM. Moreover, the two inducible lines have the advantage of strict temporal control. All conditional and conditional inducible lines were crossed with various reporter lines to mark Cre activity, discussed in detail below. We began by assessing reporter expression in the homeostatic lung.

Flow cytometry was used to assess reporter expression in cells from digested lung tissue (Figure 1A). Using surface expression of CD45, cells were split into hematopoietic $\left(\mathrm{CD} 45^{+}\right)$and non-hematopoietic $\left(\mathrm{CD} 45^{-}\right)$populations. Within non-hematopoietic cells, we identified epithelial cells as $\mathrm{EpCAM}^{+}$, and endothelial cells as $\mathrm{EpCAM}^{-}, \mathrm{CD} 1^{+}$and thrombomodulin ${ }^{+}$. To identify our populations of interest in hematopoietic cells, we first excluded neutrophils using Ly6G. Within Ly6G ${ }^{-}$cells, macrophages were identified as double-positive for CD64 and 

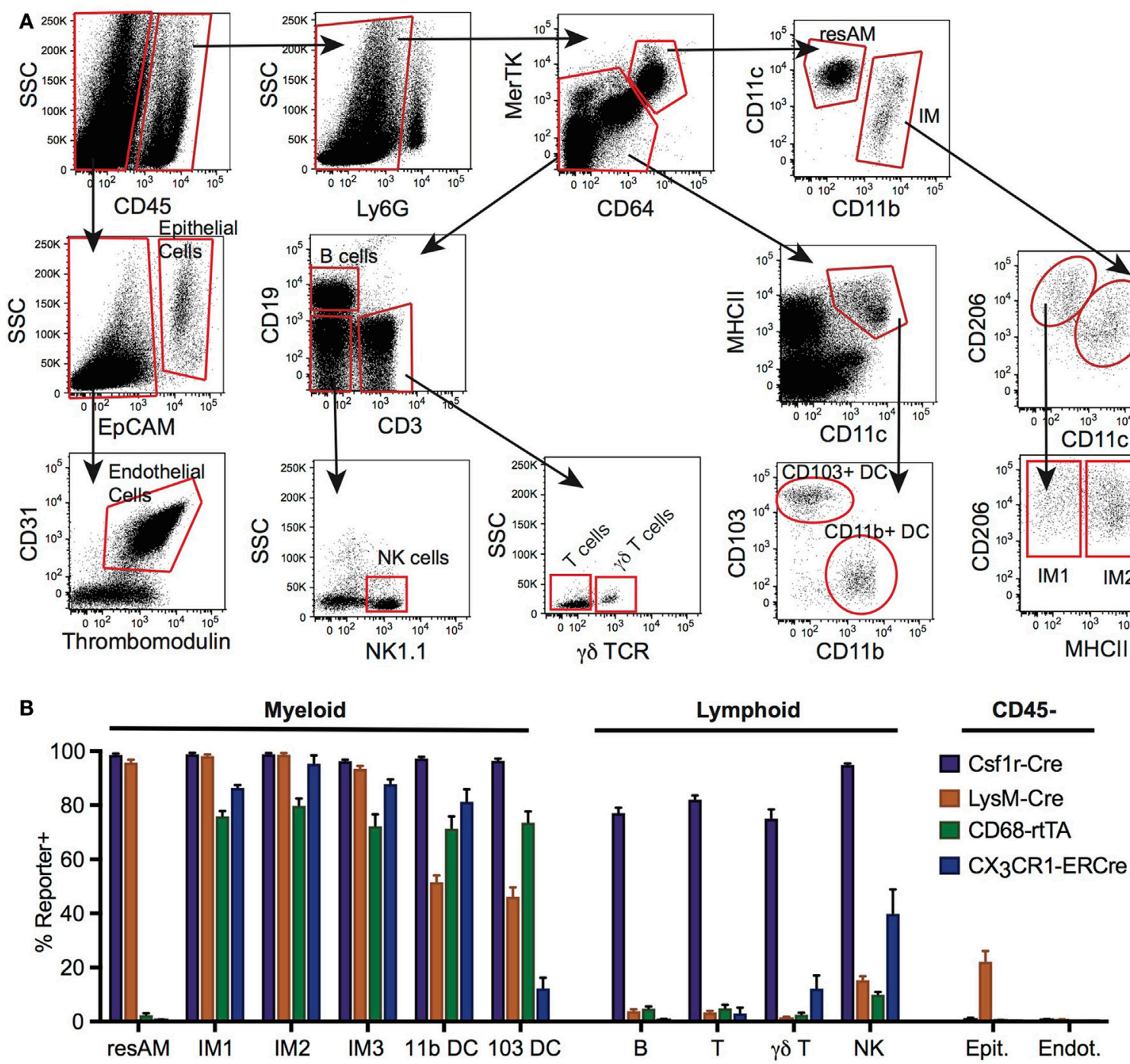

C

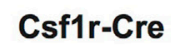

LysM-Cre
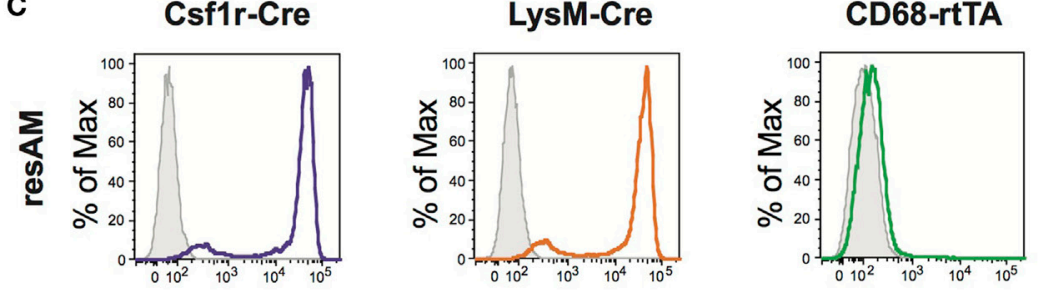

$\mathrm{CX}_{3} \mathrm{CR} 1-\mathrm{ERCre}$
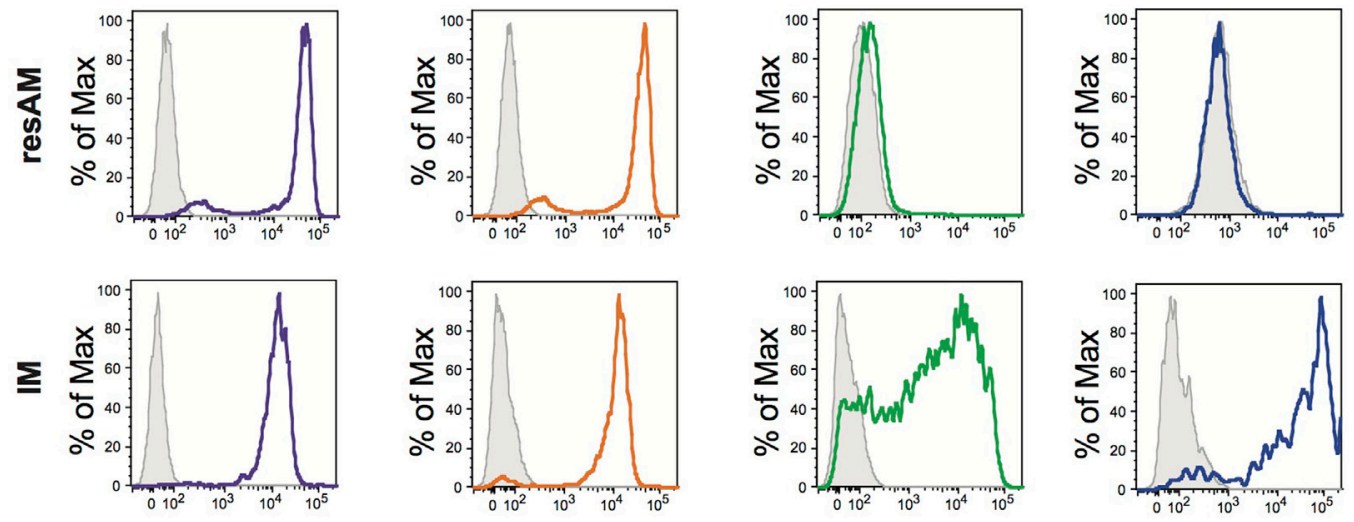

Reporter

FIGURE 1 | Conditional and inducible promoters vary in lung macrophage targeting. (A) Lung digest from reporter mice was assessed by flow cytometry and separated into subpopulations based on surface marker expression. (B) Reporter expression in lung subpopulations of myeloid, lymphoid, and structural cells, shown as percent of each population expressing the reporter. (C) Representative histograms of reporter expression in resident alveolar macrophages (ResAM) and interstitial macrophages (IM) populations. Data are presented as mean $\pm \mathrm{SD}, n=3-5$ mice per group. 
MerTK, and non-macrophages as low or negative for CD64 and MerTK expression. From the non-macrophage population, lymphocyte populations were segregated. B cells were identified by their high expression of $\mathrm{CD} 19^{+}$. $\mathrm{CD}^{+}$was used to identify T cells that were further identified as $\gamma \delta \mathrm{T}$ cells or classical $\alpha \beta$ T cells by expression of the $\gamma \delta$ TCR. Within remaining $\mathrm{CD} 19^{-} \mathrm{CD}^{-}$cells, NK cells were identified as NK1.1 $1^{+}$. Also within the non-macrophage population, CD64- and MerTK ${ }^{-}$ cells, DCs were identified by their high expression of MHCII and $\mathrm{CD} 11 \mathrm{c}$, and then further subdivided into $\mathrm{CD} 11 \mathrm{~b}^{+} \mathrm{DC}$ and $\mathrm{CD}_{103^{+}} \mathrm{DC}$ by expression of their eponymous markers. The macrophage population identified by MerTK and CD64 was divided into CD $11 c^{\text {hi }}$ ResAM and CD $11 b^{\text {hi }} C D 11 c^{\text {hi/lo }} I M s$. IMs were then divided into subsets, first using CD11c and CD206 to identify IM3 (CD206 $\left.{ }^{\text {low }}\right)$, and then remaining cells $\left(\mathrm{CD} 206^{\mathrm{hi}}\right)$ were defined as IM1 and IM2 using expression of MHCII.

To assess the efficiency and specificity of the Csf1r-Cre and LysM-Cre conditional systems, each strain was crossed with R26-stop $\mathrm{f}^{\mathrm{f} / \mathrm{fl}}$-TdTomato reporter animals. In the resultant offspring, cells that express Cre excise the floxed stop codon upstream of the TdTomato construct and the fluorescent protein is expressed. Both Csf1r-Cre and LysM-Cre were strongly activated in ResAM and IM, with nearly $100 \%$ efficiency (Figures 1B,C). However, further examination of Csf1r-Cre demonstrated that this conditional line targeted nearly all leukocytes in the lung, including lymphocytes, with greater than $80 \%$ efficiency (Figure 1B). In contrast, LysM-Cre minimally target lymphocytes, although it did target approximately half of Ly6C ${ }^{\text {hi }}$ monocytes, CD $11 b^{+}$DC and $\mathrm{CD}_{103^{+}}$DC (Figure 1B) (9). Of significant importance, LysM-Cre targeted nearly a quarter of lung epithelial cells (Figure 1B). Previous studies have suggested that these targeted epithelial cells are alveolar type II cells (1). In comparison, Csf1r-Cre did not target lung epithelium or endothelium. Data presented in Figure 1B are also summarized in Table $\mathbf{1}$ and representative histograms for all cell populations are shown in Figure S1 in Supplementary Material.

\section{Inducible Cre Systems Target Lung Macrophages with Varying Specificity}

As a next step, the inducible targeting systems were explored. First, $\mathrm{CX}_{3} \mathrm{CR} 1$-ERCre mice were crossed with R26-stop ${ }^{\mathrm{f} / \mathrm{fl}}$ TdTomato reporter mice. In these animals, cells express a mutated Cre that is directed by the $\mathrm{CX}_{3} \mathrm{CR} 1$ promoter and requires tamoxifen as a co-factor for activity. Accordingly, when tamoxifen is administered, Cre recombinase becomes active in all $\mathrm{CX}_{3} \mathrm{CR} 1$ expressing cells, thereby excising the floxed R26-stop floxed codon and enabling production of TdTomato. In a similar fashion, CD68-rtTA mice were crossed with tetOn-GFP mice. In these animals, rtTA acts as a transcription factor for the tetOn promoter, but requires the presence of doxycycline for activity. Thus, GFP is expressed in CD68 positive cells following administration of doxycycline. The duration of tamoxifen or doxycycline administration will affect the efficiency of targeting as the percentage of target cells affected increases over time. We have previously reported that CD68-rtTA reporter expression
TABLE 1 | Reporter expression in cell populations from naïve lung digest and peripheral blood.

\begin{tabular}{|c|c|c|c|c|c|}
\hline & & Csf1r-Cre & LysM-Cre & $\begin{array}{c}\mathrm{CX}_{3} \mathrm{CR} 1- \\
\text { ERCre }\end{array}$ & CD68-rtTA \\
\hline \multirow{12}{*}{$\begin{array}{l}\text { Lung } \\
\text { digest }\end{array}$} & ResAM & $98.3 \pm 0.4$ & $96.1 \pm 1.8$ & $\leq 1$ & $2.59 \pm 0.8$ \\
\hline & $\mathrm{IM} 1$ & $99.8 \pm 0.3$ & $98.5 \pm 0.8$ & $86.6 \pm 1.5$ & $76.13 \pm 3$ \\
\hline & IM2 & $99.6 \pm 0.5$ & $98.9 \pm 0.8$ & $95.7 \pm 5$ & $80 \pm 4.3$ \\
\hline & IM3 & $99.8 \pm 0.1$ & $93.7 \pm 1.9$ & $88.1 \pm 2.5$ & $72.5 \pm 7.2$ \\
\hline & CD11b+DC & $99.6 \pm 0.4$ & $52.8 \pm 3.8$ & $81.6 \pm 7.5$ & $71.6 \pm 7.3$ \\
\hline & CD103+ DC & $99.7 \pm 0.3$ & $44.8 \pm 5.5$ & $12.5 \pm 6.4$ & $73.8 \pm 6.8$ \\
\hline & T cells & $95.6 \pm 6.5$ & $5.2 \pm 1.5$ & $3.3 \pm 3.2$ & $5.1 \pm 1.8$ \\
\hline & B cells & $79 \pm 9.5$ & $5.4 \pm 1$ & $\leq 1$ & $7.4 \pm 1$ \\
\hline & $\gamma \delta \mathrm{T}$ cells & $95.1 \pm 5.2$ & $3.1 \pm 0.74$ & $5.4 \pm 1.5$ & $2.8 \pm 0.8$ \\
\hline & NK cells & $98.9 \pm 1.8$ & $15.6 \pm 2.5$ & $40.1 \pm 15.2$ & $10.2 \pm 1.3$ \\
\hline & $\begin{array}{l}\text { Endothelial } \\
\text { cells }\end{array}$ & $\leq 1$ & $\leq 1$ & $\leq 1$ & $\leq 1$ \\
\hline & Epithelial cells & $\leq 1$ & $22.3 \pm 7.3$ & $\leq 1$ & $\leq 1$ \\
\hline \multirow[t]{6}{*}{ Blood } & Neutrophils & $99.5 \pm 0.4$ & $98 \pm 1.1$ & $1.7 \pm 1.4$ & $58.7 \pm 9.7$ \\
\hline & Eosinophils & $93 \pm 5.1$ & $19.8 \pm 1.6$ & $\leq 1$ & $1.2 \pm 0.5$ \\
\hline & $\begin{array}{l}{\text { Ly } 6 C^{\text {hi }}} \\
\text { monocytes }\end{array}$ & $100 \pm 0$ & $67 \pm 4.9$ & $51 \pm 4.8$ & $70.5 \pm 6.3$ \\
\hline & $\begin{array}{l}\text { Ly6C }^{\text {lo }} \\
\text { monocytes }\end{array}$ & $99.9 \pm 0.9$ & $86.5 \pm 1.4$ & $91.5 \pm 9.1$ & $63.8 \pm 4.3$ \\
\hline & T cells & $98.5 \pm 1$ & $6.7 \pm 1.8$ & $6.9 \pm 3.3$ & $6.9 \pm 2.2$ \\
\hline & B cells & $82.5 \pm 3.5$ & $6.1 \pm 0.9$ & $\leq 1$ & $8.3 \pm 2.7$ \\
\hline
\end{tabular}

Data are presented as mean $\pm S D, n=3-5$ mice per group.

increases to a maximum level over 6 days (29). We therefore assessed $\mathrm{CX}_{3} \mathrm{CR} 1$-ERT2 and CD68-rtTA reporter mice after 1 week of tamoxifen or doxycycline.

$\mathrm{CX}_{3} \mathrm{CR} 1-\mathrm{ERCre}$ and CD68-rtTA efficiently targeted IM, with 90 and 75\% efficiency, respectively (Figures 1B,C). However, ResAM remained reporter negative in both lines, as we have shown previously for CD68-rtTA (29). In addition to IMs, both $\mathrm{CX}_{3} \mathrm{CR} 1$-ERCre and CD68-rtTA targeted lung DCs (Figure 1B). CX ${ }_{3}$ CR1-ERCre also targeted a significant portion of NK cells, although targeting of other lymphocytes remained low (Figure 1B). Reporter expression was not observed in endothelial or epithelial cells for either line. The data presented in Figure 1B is also summarized in Table $\mathbf{1}$ and representative histograms for all cell populations are shown in Figure S1 in Supplementary Material.

\section{Conditional and Inducible Cre Systems Target Blood Myeloid Cells with Varying Specificity}

During homeostasis, Ly6 $\mathrm{C}^{\text {hi }}$ monocytes constitutively traffic through the lungs (30). During inflammation, emigration of these cells from the circulation is increased and it is believed that they give rise to RecM $\Phi$. Neutrophils and other leukocytes also emigrate from the bloodstream during inflammation. Therefore, we assessed activation of Csf1r-Cre, LysM-Cre, CX ${ }_{3}$ CR1-ERCre, and CD68-rtTA in blood leukocytes. Blood was obtained by cardiac puncture, red cells were lysed, and reporter expression was measured by flow cytometry (Figure 2A). First, CD $45^{+}$ leukocytes were identified. Neutrophils were identified using 


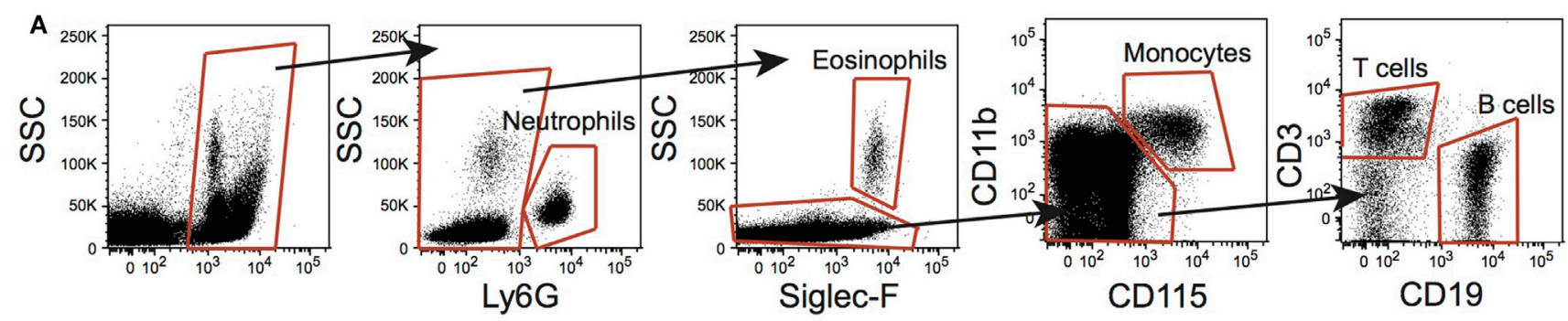

B

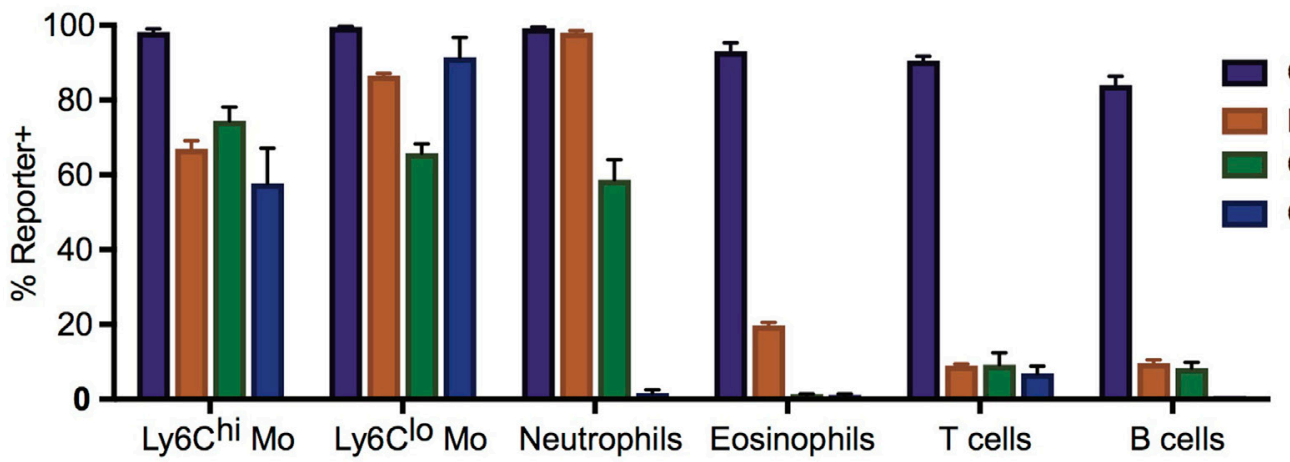

C
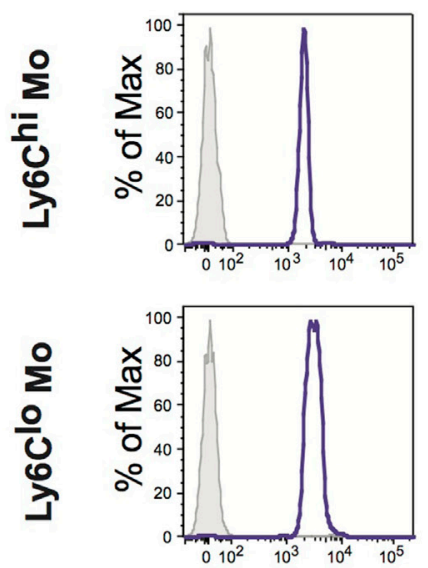

LysM-Cre
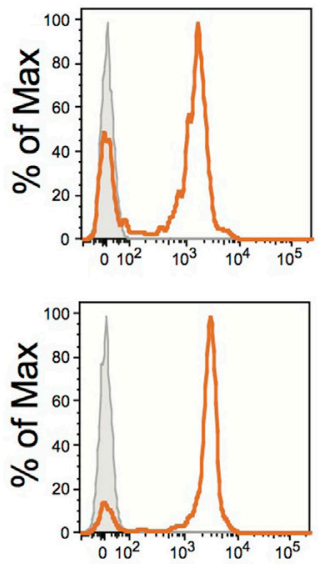

CD68-rtTA
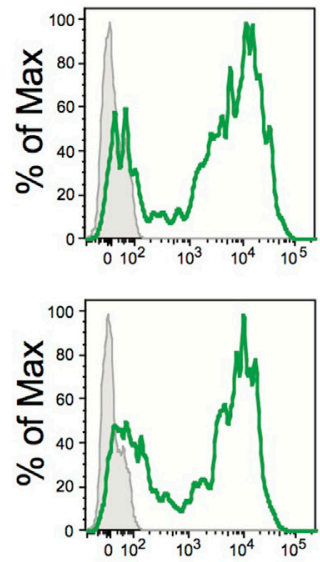

CX ${ }_{3}$ CR1-ERT2
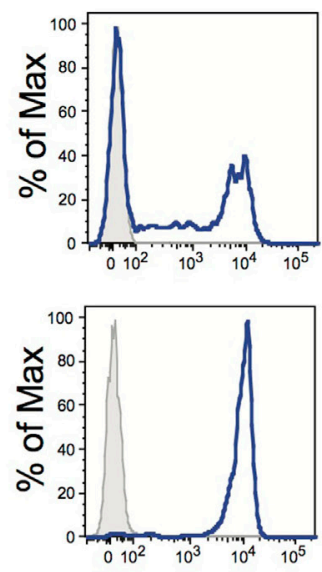

Reporter

FIGURE 2 | Conditional and inducible promoters vary in blood myeloid targeting. (A) Peripheral blood from reporter mice was assessed by flow cytometry and separated into subpopulations based on surface marker expression. (B) Reporter expression in blood subpopulations, shown as percent of each population expressing the reporter. (C) Representative histograms of reporter expression in Ly6C ${ }^{\text {hi }}$ and Ly6C $\mathrm{C}^{\mathrm{l}}$ monocyte populations. Data are presented as mean $\pm \mathrm{SD}$, $n=3-5$ mice per group.

Ly6G, and eosinophils distinguished using Siglec-F. Monocytes were identified from remaining cells as $\mathrm{CD} 115^{+} \mathrm{CD} 11 \mathrm{~b}^{+}$. B and $\mathrm{T}$ cells were identified within the remaining pool as $\mathrm{CD} 19^{+}$or $\mathrm{CD}^{+}$, respectively.

Ly6 $\mathrm{C}^{\text {hi }}$ and Ly6C $\mathrm{C}^{\text {lo }}$ monocyte subsets were targeted by all four lines, with the highest percentage of cells targeted by Csf1r-Cre (Figures 2B,C). Csf1r-Cre, LysM-Cre, and CD68-rtTA were activated in neutrophils, and both Csf1r-Cre and LysM-Cre were activated in eosinophils (Figure 2B). Only Csf1r-Cre was completely activated in lymphocytes (Figure 2B). The data presented in Figure 2B is also summarized in Table $\mathbf{1}$ and representative histograms for all cell populations are shown in Figure S2 in Supplementary Material.

Although resident tissue macrophages of other organs do not directly migrate to the lung, macrophages of other organs, particularly the bone marrow and spleen, regulate hematopoiesis, and impact circulating leukocyte levels (31-33). We examined reporter expression of Csf1r-Cre, LysM-Cre, $\mathrm{CX}_{3} \mathrm{CR} 1$-ERCre, and CD68-rtTA-driven lines in bone marrow, spleen, and PL using flow cytometry (Figures S3A,B,E in Supplementary Material). Macrophages in all three tissues were targeted by all four drivers (Figures S3C,D,F,G in Supplementary Material). 


\section{Timing of Inducible Cre Systems Can Be Used to Refine Specificity for Lung IM}

A distinct advantage of inducible Cre systems such as $\mathrm{CX}_{3} \mathrm{CR} 1-$ ERCre and CD68-rtTA is that the timing and duration of tamoxifen or doxycycline administration can be used to enhance cell selectivity and specificity. As an example, we explored the kinetics of dosing in both inducible strains to refine targeting of IM. Mice were treated with either tamoxifen or doxycycline for
1 week, then the drug was withdrawn and reporter expression was examined 1,2 , and 4 weeks later.

In the lung tissue of both $\mathrm{CX}_{3} \mathrm{CR} 1-\mathrm{ERCre}$ and CD68-rtTA mice, IM1 and IM2 reporter expression remained stable after tamoxifen or doxycycline was withdrawn, with only a $5 \%$ decrease in reporter positive cells over 4 weeks (Figures 3A,B). IM3 showed a steeper rate of declining reporter expression, with a $20 \%$ decrease in reporter positive cells (Figures $\mathbf{3 A , B}$ ).

\section{3 CR1-ERCre}
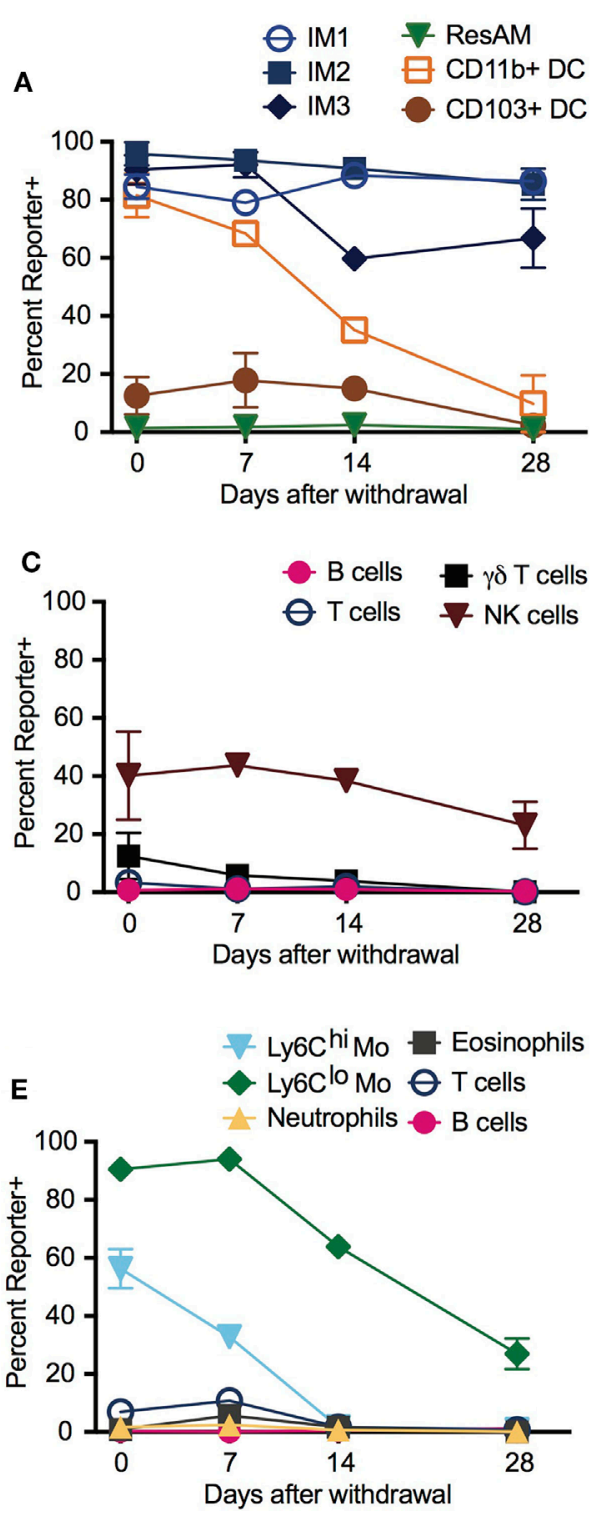

CD68-rtTA

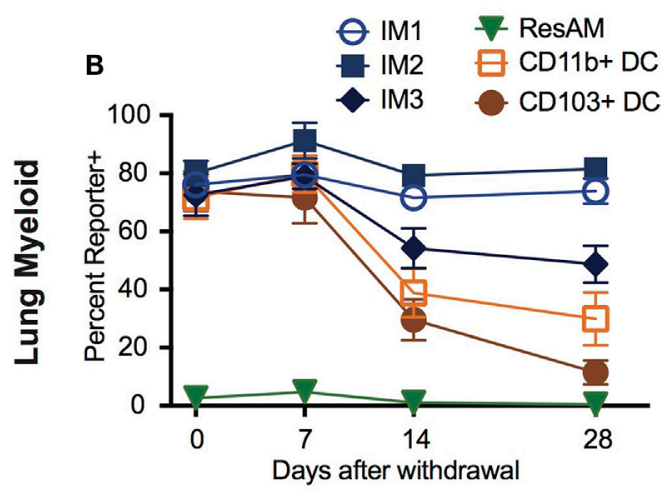

D
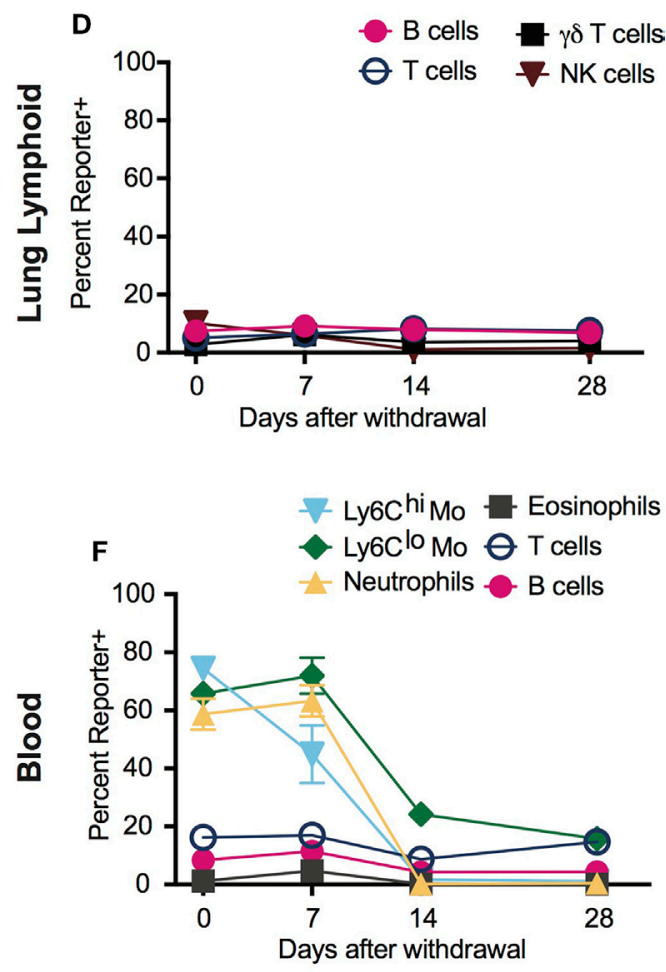

FIGURE 3 | Dosing of tamoxifen and doxycycline can be manipulated to enhance targeting of interstitial macrophages (IMs) in inducible systems. CX ${ }_{3}$ CR1-estrogen receptor-Cre (ERCre) and CD68-rtTA reporter mice were administered tamoxifen or doxycycline, respectively, for 1 week, then administration was halted and mice were examined 1,2, and 4 weeks later. (A) Reporter expression in lung myeloid cells from $\mathrm{CX}_{3} \mathrm{CR} 1$-ERCre mice over time after withdrawal from tamoxifen. (B) Reporter expression in lung myeloid cells from CD68-rtTA mice over time after withdrawal from doxycycline. (C) Reporter expression in lung lymphoid cells from $\mathrm{CX}_{3} \mathrm{CR} 1$-ERCre mice over time after withdrawal from tamoxifen. (D) Reporter expression in lung lymphoid cells from CD68-rtTA mice over time after withdrawal from doxycycline. (E) Reporter expression in peripheral blood cells from $\mathrm{CX}_{3} \mathrm{CR} 1$-ERCre mice over time after withdrawal from tamoxifen. (F) Reporter expression peripheral blood cells from CD68-rtTA mice over time after withdrawal from doxycycline. Data are presented as mean \pm SD, $n=3-5$ mice per group. 
As anticipated, ResAM were reporter negative at all times. Importantly, the percentage of reporter positive DC dropped to less than $10 \%$ in $\mathrm{CX}_{3} \mathrm{CR} 1$-ERCre mice by 4 weeks and to less than $30 \%$ in CD68-rtTA mice. Reporter expression was similarly low in lymphocytes isolated from the lungs, with the exception of NK cells in $\mathrm{CX}_{3} \mathrm{CR} 1$-ERCre mice, in which nearly $25 \%$ remained positive (Figures 3C,D).

Reporter expression in neutrophils and monocytes from peripheral blood was also lost after 4 weeks of tamoxifen or doxycycline withdrawal (Figures 3E,F). Virtually, all of the neutrophils and Ly6C ${ }^{\text {hi }}$ monocytes were TdTomato reporter negative, whereas approximately $20 \%$ of Ly6C ${ }^{\text {lo }}$ monocytes remained TdTomato positive in both strains. These findings support the concept that neutrophils and Ly6C hi monocytes have relatively short lifespans, and that turnover of neutrophils and Ly $6 \mathrm{C}^{\text {hi }}$ monocytes are relatively high (30). In addition, since excision of floxed alleles is permanent in affected cells (i.e., cells stay reporter positive for life), the observation that the IM3 population gradually loses reporter expression suggests that at least a portion of these cells are replaced by monocyte precursors, supporting previous reports using parabiotic mice (14). Taken together, these findings show that dosing of tamoxifen and doxycycline can be manipulated to enhance targeting of IMs in CD68-rtTA and $\mathrm{CX}_{3}$ CR1-ERCre mice.

\section{Conditional Cre Systems Target Recruited Alveolar Macrophages following LPS Injury}

Inflammation has been reported to alter Cre activity, and during inflammation a new population of macrophages is present in the lung airways: RecM $\Phi$. This macrophage population is believed to arise from infiltrating monocytes maturing in situ, and can be distinguished from ResAM by high expression of CD11b (Figure 4A) [and low expression of Siglec-F $(17,34)$ ]. We assessed Csf1r-Cre and LysM-Cre reporter mice following LPS injury for the potential changes to Cre activity and measured reporter expression in recruited macrophages. RecM $\Phi$ were targeted by both Csf1r-Cre and LysM-Cre with near 100\% efficiency (Figures 4B-D). Efficiency for all three IM populations was also high (>95\%; Table 2). However, as seen during homeostasis, other leukocyte populations were also highly targeted in Csf1r-Cre mice and to a lesser extent in LysM-Cre animals (Table 2).

\section{ResAM Are Resistant to rtTA-Driven Doxycycline-Induced Transgene Expression}

We have previously reported that ResAM do not activate CD68-rtTA in vivo after 21 days of doxycycline, or in vitro when doxycycline was provided in culture media for 4 days (29). However, it was unclear whether this restriction reflected a failure to activate the CD68 promoter or resulted from decreased rtTA activity. To assess this, we obtained R26-rtTA mice (35), where rtTA is expressed under control of the Rosa26 promoter, which is ubiquitously expressed by all leukocytes including ResAM (36). Both CD68-rtTA and R26-rtTA mice were crossed with tetOn-GFP mice, and resulting offspring were administered doxycycline for 10 months. Notably, ResAM from CD68-rtTA mice remained GFP negative, although the majority of myeloid cells (including IM) were GFP positive

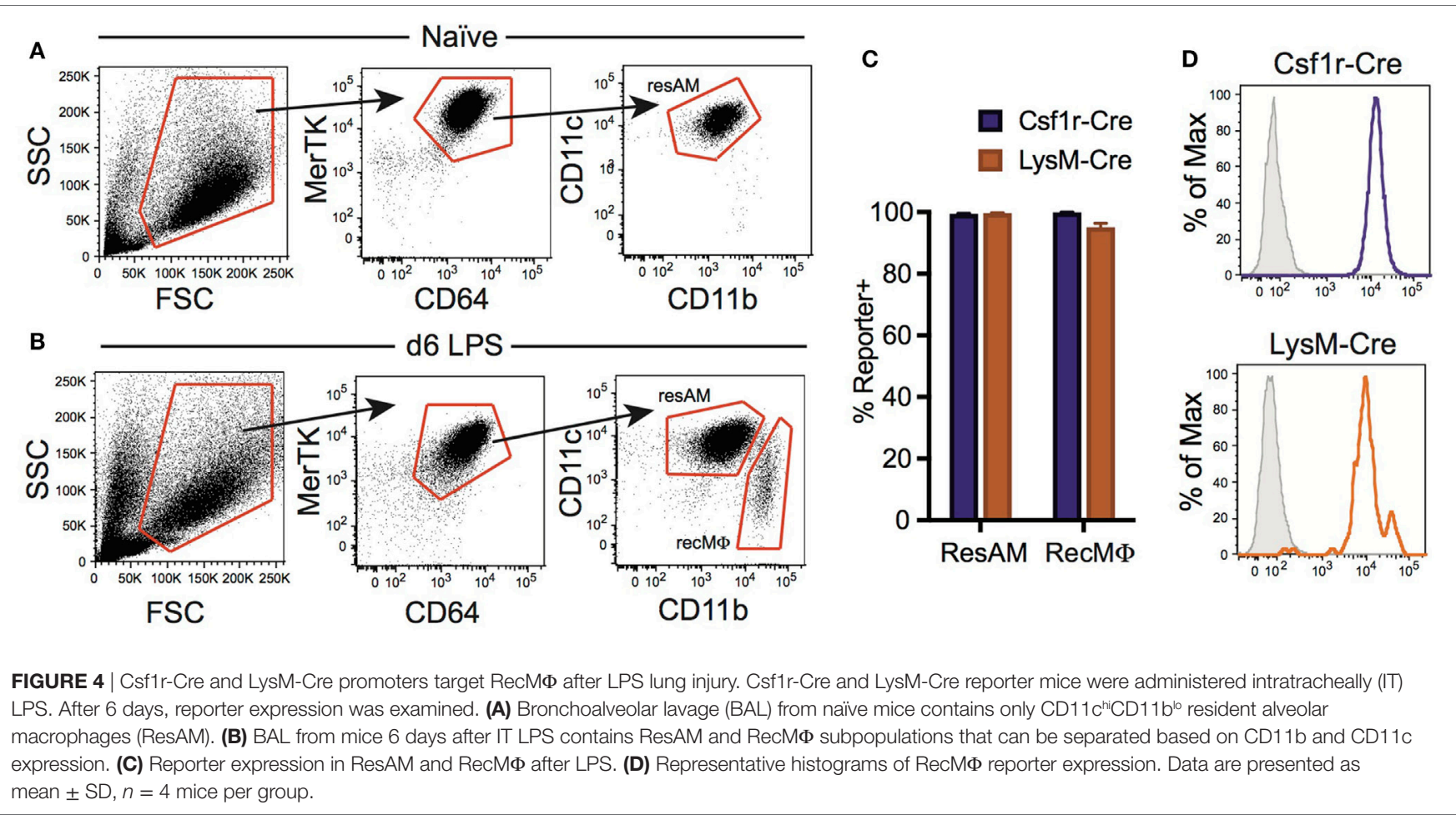


TABLE 2 | Reporter expression in cell populations from bronchoalveolar lavage (BAL), lung digest, and peripheral blood 6 days after intratracheally LPS.

\begin{tabular}{|c|c|c|c|}
\hline & & Csf1r-Cre & LysM-Cre \\
\hline \multirow[t]{2}{*}{$\mathrm{BAL}$} & ResAM & $98.9 \pm 0.7$ & $96.6 \pm 0.9$ \\
\hline & $\operatorname{RecM\Phi }$ & $99.9 \pm 0.2$ & $95.2 \pm 2.5$ \\
\hline \multirow[t]{9}{*}{ Lung digest } & $\mathrm{IM} 1$ & $96.6 \pm 0.7$ & $90.2 \pm 2.7$ \\
\hline & IM2 & $98.2 \pm 0.8$ & $93.9 \pm 0.5$ \\
\hline & IM3 & $98.9 \pm 0.6$ & $93.5 \pm 0.9$ \\
\hline & CD11b+ DC & $98.6 \pm 0.9$ & $53.6 \pm 4.4$ \\
\hline & CD103+ DC & $98.4 \pm 0.6$ & $48.4 \pm 6.8$ \\
\hline & T cells & $91.9 \pm 1.4$ & $3.1 \pm 0.7$ \\
\hline & B cells & $79.9 \pm 6.8$ & $5.2 \pm 0.8$ \\
\hline & $\gamma \delta \mathrm{T}$ cells & $91.5 \pm 2.3$ & $3.4 \pm 1.1$ \\
\hline & NK cells & $97.6 \pm 0.5$ & $14.7 \pm 1.1$ \\
\hline \multirow[t]{6}{*}{ Blood } & Neutrophils & $99.8 \pm 0.2$ & $98.9 \pm 0.5$ \\
\hline & Eosinophils & $98.8 \pm 0.4$ & $12.5 \pm 0.5$ \\
\hline & Ly6C $C^{\text {hi }}$ monocytes & $99.9 \pm 0.3$ & $78.2 \pm 1.7$ \\
\hline & Ly6C $C^{10}$ monocytes & $99.9 \pm 0.2$ & $88.1 \pm 1.0$ \\
\hline & T cells & $99.8 \pm 0.1$ & $4.2 \pm 0.8$ \\
\hline & B cells & $87.8 \pm 4.2$ & $2.9 \pm 0.4$ \\
\hline
\end{tabular}

Data are presented as mean $\pm S D, n=4$ mice per group.

(Figures 5A,B). Strikingly, ResAM from R26-rtTA mice also failed to express GFP, whereas $>95 \%$ of other leukocytes were GFP positive (Figures $\mathbf{5 A}, \mathbf{C}$ ). We conclude that ResAM are resistant to the activation of rtTA-driven systems. Accordingly, the CD68-rtTA system can be exploited to target-specific lung tissue macrophage populations and to distinguish the roles they play during both homeostasis and in lung disease.

\section{DISCUSSION}

Cre-LoxP strategies are commonly employed to delete genes in specific cell types or tissues. However, accurate data interpretation requires a firm understanding of the underlying genetic principles and detailed knowledge regarding the specificity and efficiency of the Cre driver. To this end, we assessed the operating characteristics of four commonly used and commercially available mouse lines that are published as "macrophage targeting": Csf1r-Cre, LysM-Cre, CX 3 CR1-ERCre, and CD68-rtTA. We found that each line targeted multiple non-macrophage populations in the lungs and circulation. In conditional deletion systems, LysM-Cre had greater specificity for lung macrophages than Csf1r-Cre but also targeted type II alveolar cells, and was less efficient at targeting monocytes. The $\mathrm{CX}_{3} \mathrm{CR} 1$-ERCre and CD68-rtTA systems robustly targeted IMs and trafficking monocytes, but did not delete floxed genes in resident AMs. Non-macrophage populations were also targeted in these inducible systems; however, macrophage specificity was improved once tamoxifen or doxycycline was withdrawn. As discussed below, these findings can be used to one's advantage and can enable time and cell-specific targeting of specific lung macrophage populations.

It is critical to appreciate that in conditional and inducible Cre lines, once a cell activates the driver promoter, floxed DNA sequences will be permanently deleted from that time forward-even if the driver promoter is later downregulated.

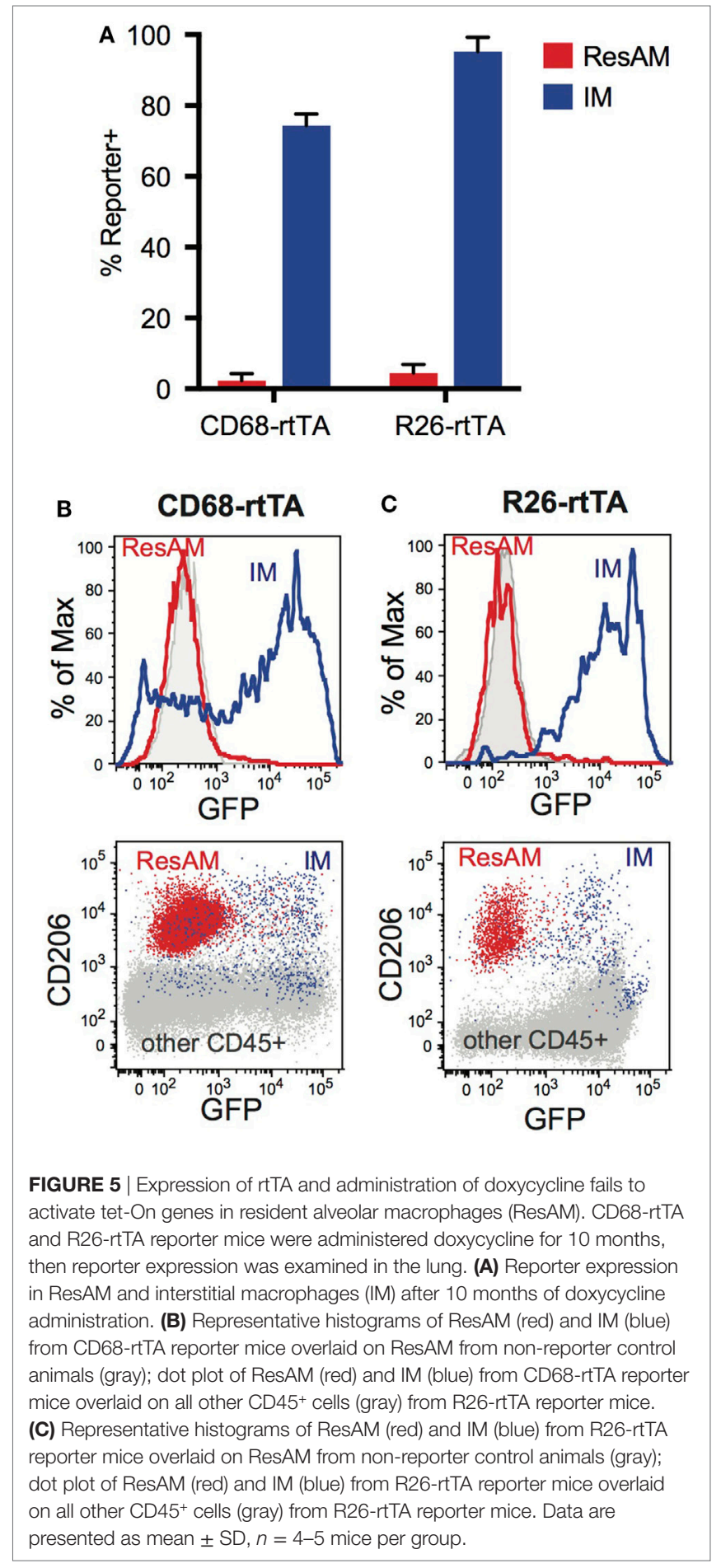

Moreover, the floxed sequences will also be permanently deleted in all daughter cells. This point can be best illustrated by comparing $\mathrm{CX}_{3} \mathrm{CR} 1$-Cre mice (11) (not studied herein) to $\mathrm{CX}_{3} \mathrm{CR} 1$-ERCre mice. In conditional $\mathrm{CX}_{3} \mathrm{CR} 1$-Cre mice, floxed alleles are deleted in ResAM because the precursors for ResAM express $\mathrm{CX}_{3} \mathrm{CR} 1$ during embryogenesis. However, in the inducible $\mathrm{CX}_{3} \mathrm{CR} 1$-ERCre mice ResAM are not targeted when tamoxifen is administered to adult mice because mature ResAM 
do not express $\mathrm{CX}_{3} \mathrm{CR} 1$ and are not replaced by circulating monocytes (11).

Importantly, the cell specificity of Cre-LoxP strategies may differ from that of non-Cre reporter systems. For instance, in our studies the Csf1r-Cre system affects almost all leukocytes. In comparison, when the Csflr promoter is used to directly drive a fluorescent reporter [as in the Mafia mouse (37)], the fluorescent protein is only expressed when the Csf1r promoter is actively engaged. When promoter engagement ceases, GFP only remains until it is degraded, or until the cell undergoes sufficient divisions to dilute the signal. Accordingly, labeling is primarily restricted to myeloid cells in the Mafia mouse, even though the promoter is the same as in Csflr-Cre animals. We speculate that early, irreversible recombination in hematopoietic precursor cells is responsible for the non-specific Cre activation in Csf1rCre mice even though in mature leukocytes Csflr expression is restricted to myeloid cells.

The four Cre driver lines that we tested each have positives and negatives for their use. Csf1r-Cre targets ResAM and IM with the highest efficiency, but the lowest specificity. All leukocytes examined were targeted by Csf1r-Cre, although Csf1r-Cre did not target lung epithelium or endothelium. In contrast, nearly a quarter of lung epithelium was targeted by LysM-Cre. ResAM and IM were also targeted with high efficiency by LysM-Cre, but neutrophils, DC, and monocytes were affected as well. $\mathrm{CX}_{3} \mathrm{CR} 1$-ERCre targeted IM with high efficiency but did not target ResAM. $\mathrm{CX}_{3}$ CR1-ERCre also targeted CD11 $\mathrm{b}^{+}$ DC, Ly6 $\mathrm{C}^{\mathrm{lo}}$ monocytes, and NK cells, but did not affect other cell populations including lung endothelial and epithelial cells. Off-target effects on non-macrophage populations decreased once tamoxifen was withdrawn as $\mathrm{CD} 11 \mathrm{~b}^{+} \mathrm{DC}$, monocytes, and NK cells were replaced by unaffected precursors. CD68rtTA targeted IM with moderate efficiency, and did not target ResAM. CD68-rtTA also targeted DC, monocytes, and neutrophils, but did not affect lymphocytes, endothelial, or epithelial cells. As seen with $\mathrm{CX}_{3}$ CR1-ERCre, off-target effects on non-macrophage populations decreased once doxycycline was withdrawn as DC, monocytes, and neutrophils were replaced by unaffected precursors.

Inducible $\mathrm{CX}_{3} \mathrm{CR} 1$-ERCre and CD68-rtTA mice provide an opportunity to discretely target IM without affecting ResAM. IM have been far less studied then ResAM due to the difficult nature of their isolation and their low numbers compared with ResAM. Hence, since the inducible model systems described herein uniquely target IM, these provide a critical advance for their study. Notably, the $\mathrm{CX}_{3} \mathrm{CR} 1$-ERCre system targets IM with slightly greater efficiency than CD68-rtTA and targets fewer DC, and no neutrophils, suggesting this may be the superior model. However, $\mathrm{CX}_{3} \mathrm{CR} 1$-ERCre affects NK cells, which is not observed in CD68-rtTA mice. Accordingly, both models represent powerful tools for the study of lung IM, especially when deletion of floxed alleles in ResAM is not desirable.

While the biologic mechanism that restricts $\mathrm{CX}_{3} \mathrm{CR} 1$-ERCre targeting in ResAM is clear (as discussed above), the failure of CD68-rtTA to target ResAM is less apparent since mature ResAM express high levels of CD68 $(14,29)$. To this end, we examined activation of the R26-rtTA line in ResAM as a comparator. Like
CD68, endogenous R26 protein is expressed by mature ResAM. However, R26-rtTA was not activated in ResAM. We therefore suggest that ResAM may not be amenable to targeting with doxycycline-inducible systems. Although not tested here, we speculate that ResAM have the ability to export doxycycline from the cell, thereby preventing its action as a co-factor in the nucleus. Notably, ResAM uniquely express high levels of ABCG1, a homolog for bacterial transporters of tetracyclines. This does not diminish the utility of CD68-rtTA for lung studies, but should caution investigators about attempting to use any rtTA system to target ResAM.

Although we have identified two viable lines for the study of IM, none of the lines examined in this paper specifically target ResAM. CD11c-Cre has been reported to target ResAM, although careful studies of its specificity and efficiency have not been published. Since CD11c is expressed by DC, trafficking monocytes in tissue, some IM, and some lymphocytes (particularly NK cells), we expect that its targeting would be non-specific. Mafb-Cre provides an alternative possibility for targeting ResAM because it is required for the differentiation of monocytes and macrophages, but suppresses DC and granulocyte differentiation $(38,39)$. Although Mafb expression in mature ResAM is very low, ResAM are targeted by Mafb-Cre $(14,39)$ presumably due to activation in precursors. Additionally, Mafb-Cre minimally affects DC or granulocytes, and although bulk monocytes express Mafb, reporter mice showed that Mafb-Cre targeted only $10 \%$ of Ly $6 \mathrm{C}^{\text {hi }}$ and $40 \%$ of Ly6 $\mathrm{C}^{\text {lo }}$ monocytes (39). RecM $\Phi$ in the lung following exposure to house dust mite antigen are targeted by Mafb-Cre (39). IM targeting has not been assessed, although we would expect IM to be efficiently targeted. Targeting of B cells was found to be very low (39), although targeting of other lymphocytes or lung epithelial cells was not reported. The lack of DC and granulocyte targeting makes Mafb-Cre a promising line for discrete targeting of macrophages. Both CD11c-Cre and Mafb-Cre are commercially available. However, both CD11c-Cre and MafbCre will target macrophages in many organs, not specifically lung macrophages, as we observed for Csf1r-Cre, LysM-Cre, $\mathrm{CX}_{3} \mathrm{CR} 1-\mathrm{ERCre}$, and CD68-rtTA. In addition, Mafb-Cre will not discriminate gene deletion between ResAM and IM in the lung. Lack of organ specificity is another limiting factor of "macrophage specific" conditional and inducible systems. We hypothesize a tamoxifen-inducible Siglec-F-ERCre might overcome this limitation and provide a lung ResAM-specific inducible system. ResAM are the only tissue macrophage known to express Siglec-F $(34,40)$. Siglec-F is not expressed by DC or monocytes, although it is expressed by granulocytes, particularly eosinophils. Theoretically, a pulse-wait of tamoxifen should target ResAM while targeted granulocytes turnover and are replaced from untargeted precursors. To our knowledge, no Siglec-F-driven lines have been developed.

The use of animal models remains a powerful tool for understanding the homeostatic functions of lung macrophages and their roles in pulmonary disease. While useful, Cre-LoxP systems are imperfect, and understanding their limitations is necessary for effective use. With a firm understanding of the populations targeted by each driver, vagaries of the system may even be 
harnessed to one's advantage, such as in the case of CD68-rtTA mice being used to target IM without targeting ResAM. In general, neither Csf1r-Cre nor LysM-Cre are specific for macrophagesa problem for their use in the study of lung macrophages. Inducible $\mathrm{CX}_{3} \mathrm{CR} 1-\mathrm{ERCre}$ and CD68-rtTA systems show increased specificity for macrophages, particularly after pulse-wait of tamoxifen or doxycycline, however, they only target IM and not ResAM. Ultimately, each investigator must choose for themselves which system introduces the fewest confounding factors for their research. The data provided herein aim to assist with that decision, but there is clear room for improvement in developing new lines for conditional targeting of lung macrophages.

\section{ETHICS STATEMENT}

This study was carried out in accordance with the recommendations of the Institutional Animal Care and Use Committee at National Jewish Health. The protocol was approved by the Institutional Animal Care and Use Committee at National Jewish Health (approval \#AS2574-01-20).

\section{AUTHOR CONTRIBUTIONS}

AM, WJ, and CJ conceived of the study; AM and KA executed experiments; AM, WJ, CJ, and AL-S prepared the manuscript; all authors provided intellectual input, critical feedback, discussed results, and designed experiments.

\section{FUNDING}

This study was funded by NIH: R01 HL109517, R01 HL130938 (WJ), T32 HL007085-42 (AM), R01 HL115334 (CJ).

\section{REFERENCES}

1. Miyake Y, Kaise H, Isono K, Koseki H, Kohno K, Tanaka M. Protective role of macrophages in noninflammatory lung injury caused by selective ablation of alveolar epithelial type II cells. J Immunol (2007) 178:5001-9. doi:10.4049/ jimmunol.178.8.5001

2. Abram CL, Roberge GL, Hu Y, Lowell CA. Comparative analysis of the efficiency and specificity of myeloid-Cre deleting strains using ROSAEYFP reporter mice. J Immunol Methods (2014) 408:89-100. doi:10.1016/j. jim.2014.05.009

3. Garbi N, Lambrecht BN. Location, function, and ontogeny of pulmonary macrophages during the steady state. Pflugers Arch (2017) 469:561-72. doi:10.1007/s00424-017-1965-3

4. Sauer B, Henderson N. Site-specific DNA recombination in mammalian cells by the Cre recombinase of bacteriophage P1. Proc Natl Acad Sci U S A (1988) 85:5166-70. doi:10.1073/pnas.85.14.5166

5. Lewandoski M. Conditional control of gene expression in the mouse. Nat Rev Genet (2001) 2:743-55. doi:10.1038/35093537

6. Feil S, Valtcheva N, Feil R. Inducible Cre mice. Methods Mol Biol (2009) 530:343-63. doi:10.1007/978-1-59745-471-1_18

7. Gossen M, Freundlieb S, Bender G, Muller G, Hillen W, Bujard H. Transcriptional activation by tetracyclines in mammalian cells. Science (1995) 268:1766-9. doi:10.1126/science.7792603

8. Koponen JK, Kankkonen H, Kannasto J, Wirth T, Hillen W, Bujard H, et al. Doxycycline-regulated lentiviral vector system with a novel reverse transactivator rtTA2S-M2 shows a tight control of gene expression in vitro and in vivo. Gene Ther (2003) 10:459-66. doi:10.1038/sj.gt. 3301889

\section{SUPPLEMENTARY MATERIAL}

The Supplementary Material for this article can be found online at http://www.frontiersin.org/article/10.3389/fimmu.2017.01618/ full\#supplementary-material.

FIGURE S1 | Representative histograms of reporter expression in lung cell populations from reporter lines. For each lung cell population assessed in Figure 1, representative histograms of reporter expression are shown from Csf1r-Cre, LysM-Cre, $\mathrm{CX}_{3} \mathrm{CR} 1$-estrogen receptor-Cre (ERCre), and CD68-rtTA reporter mice. $\mathrm{CX}_{3} \mathrm{CR} 1$-ERCre and CD68-rtTA data are from mice administered tamoxifen or doxycycline for 1 week. Gray histogram shows florescence of cells from non-reporter control mice.

FIGURE S2 | Representative histograms of reporter expression in peripheral blood populations from reporter lines. For each peripheral blood population assessed in Figure 2, representative histograms of reporter expression are shown from Csf1r-Cre, LysM-Cre, $\mathrm{CX}_{3} \mathrm{CR} 1$-estrogen receptor-Cre (ERCre), and CD68-rtTA reporter mice. $\mathrm{CX}_{3} \mathrm{CR} 1$-ERCre and CD68-rtTA data are from mice administered tamoxifen or doxycycline for 1 week. Gray histogram shows florescence of cells from non-reporter control mice.

FIGURE S3 | Conditional and inducible promoters vary in targeting bone marrow, splenic, and peritoneal macrophages. (A) Spleen from reporter mice was assessed by flow cytometry and separated into subpopulations based on surface marker expression. (B) Bone marrow from reporter mice was assessed by flow cytometry and separated into subpopulations based on surface marker expression. (C) Reporter expression in splenic and bone marrow

$\mathrm{CD} 11 \mathrm{~b}^{+} \mathrm{CD} 11 \mathrm{c}^{+} \mathrm{Gr}-1^{-}$macrophages, shown as percent of cells expressing the reporter. (D) Representative histograms of reporter expression of

$\mathrm{CD} 11 \mathrm{~b}^{+} \mathrm{CD} 11 \mathrm{c}^{+} \mathrm{Gr}-1^{-}$splenic and bone marrow macrophages. (E) Peritoneal lavage from reporter mice was assessed by flow cytometry and separated into subpopulations based on surface marker expression. (F) Reporter expression of Tim4 ${ }^{\text {hi }}$ and Tim4 ${ }^{\text {lo }}$ peritoneal macrophages, shown as percent of cells expressing the reporter. (G) Representative histograms of reporter expression in peritoneal macrophages. Resident peritoneal macrophages were not observed in lavage following IP tamoxifen, therefore no data are shown for peritoneal macrophages from $\mathrm{CX}_{3} \mathrm{CR} 1$-estrogen receptor-Cre mice. Data are presented as mean $\pm \mathrm{SD}$, $\mathrm{n}=3-5$ mice per group.

9. Jakubzick C, Bogunovic M, Bonito AJ, Kuan EL, Merad M, Randolph GJ. Lymph-migrating, tissue-derived dendritic cells are minor constituents within steady-state lymph nodes. J Exp Med (2008) 205:2839-50. doi:10.1084/ jem. 20081430

10. Guilliams M, De Kleer I, Henri S, Post S, Vanhoutte L, De Prijck S, et al. Alveolar macrophages develop from fetal monocytes that differentiate into long-lived cells in the first week of life via GM-CSF. J Exp Med (2013) 210:1977-92. doi:10.1084/jem.20131199

11. Yona S, Kim KW, Wolf Y, Mildner A, Varol D, Breker M, et al. Fate mapping reveals origins and dynamics of monocytes and tissue macrophages under homeostasis. Immunity (2013) 38:79-91. doi:10.1016/j.immuni.2012. 12.001

12. Sheng J, Ruedl C, Karjalainen K. Most tissue-resident macrophages except microglia are derived from fetal hematopoietic stem cells. Immunity (2015) 43:382-93. doi:10.1016/j.immuni.2015.07.016

13. Scott CL, Henri S, Guilliams M. Mononuclear phagocytes of the intestine, the skin, and the lung. Immunol Rev (2014) 262:9-24. doi:10.1111/imr. 12220

14. Gibbings SL, Thomas SM, Atif SM, Mccubbrey AL, Desch AN, Danhorn T, et al. Three unique interstitial macrophages in the murine lung at steady state. Am J Respir Cell Mol Biol (2017) 57:66-76. doi:10.1165/rcmb.20160361OC

15. Desch AN, Henson PM, Jakubzick CV. Pulmonary dendritic cell development and antigen acquisition. Immunol Res (2013) 55:178-86. doi:10.1007/ s12026-012-8359-6

16. Desch AN, Gibbings SL, Clambey ET, Janssen WJ, Slansky JE, Kedl RM, et al. Dendritic cell subsets require cis-activation for cytotoxic CD8 T-cell induction. Nat Commun (2014) 5:4674. doi:10.1038/ncomms5674 
17. McCubbrey AL, Barthel L, Mohning MP, Redente EF, Mould KJ, Thomas SM, et al. Deletion of c-FLIP from CD11bhi macrophages prevents development of bleomycin-induced lung fibrosis. Am J Respir Cell Mol Biol (2017). doi:10.1165/rcmb.2017-0154OC

18. Janssen WJ, Barthel L, Muldrow A, Oberley-Deegan RE, Kearns MT, Jakubzick C, et al. Fas determines differential fates of resident and recruited macrophages during resolution of acute lung injury. Am J Respir Crit Care Med (2011) 184:547-60. doi:10.1164/rccm.201011-18910C

19. Mould KJ, Barthel L, Mohning MP, Thomas SM, Mccubbrey AL, Danhorn T, et al. Cell origin dictates programming of resident versus recruited macrophages during acute lung injury. Am J Respir Cell Mol Biol (2017) 57:294-306. doi:10.1165/rcmb.2017-0061OC

20. Okuma T, Terasaki Y, Kaikita K, Kobayashi H, Kuziel WA, Kawasuji M, et al. C-C chemokine receptor 2 (CCR2) deficiency improves bleomycininduced pulmonary fibrosis by attenuation of both macrophage infiltration and production of macrophage-derived matrix metalloproteinases. J Pathol (2004) 204:594-604. doi:10.1002/path.1667

21. Ginhoux F, Jung S. Monocytes and macrophages: developmental pathways and tissue homeostasis. Nat Rev Immunol (2014) 14:392-404. doi:10.1038/ nri3671

22. Venosa A, Malaviya R, Gow AJ, Hall L, Laskin JD, Laskin DL. Protective role of spleen-derived macrophages in lung inflammation, injury, and fibrosis induced by nitrogen mustard. Am J Physiol Lung Cell Mol Physiol (2015) 309:L1487-98. doi:10.1152/ajplung.00276.2015

23. Misharin AV, Morales-Nebreda L, Mutlu GM, Budinger GR, Perlman H. Flow cytometric analysis of macrophages and dendritic cell subsets in the mouse lung. Am J Respir Cell Mol Biol (2013) 49:503-10. doi:10.1165/ rcmb.2013-0086MA

24. Zaynagetdinov R, Sherrill TP, Kendall PL, Segal BH, Weller KP, Tighe RM, et al. Identification of myeloid cell subsets in murine lungs using flow cytometry. Am J Respir Cell Mol Biol (2013) 49:180-9. doi:10.1165/rcmb.20120366MA

25. Deng L, Zhou JF, Sellers RS, Li JF, Nguyen AV, Wang Y, et al. A novel mouse model of inflammatory bowel disease links mammalian target of rapamycin-dependent hyperproliferation of colonic epithelium to inflammation-associated tumorigenesis. Am J Pathol (2010) 176:952-67. doi:10.2353/ ajpath.2010.090622

26. Clausen BE, Burkhardt C, Reith W, Renkawitz R, Forster I. Conditional gene targeting in macrophages and granulocytes using LysMcre mice. Transgenic Res (1999) 8:265-77. doi:10.1023/A:1008942828960

27. Pillai MM, Hayes B, Torok-Storb B. Inducible transgenes under the control of the hCD68 promoter identifies mouse macrophages with a distribution that differs from the F4/80- and CSF-1R-expressing populations. Exp Hematol (2009) 37:1387-92. doi:10.1016/j.exphem.2009.09.003

28. Hume DA. Applications of myeloid-specific promoters in transgenic mice support in vivo imaging and functional genomics but do not support the concept of distinct macrophage and dendritic cell lineages or roles in immunity. J Leukoc Biol (2011) 89:525-38. doi:10.1189/jlb.0810472

29. McCubbrey AL, Barthel L, Mould KJ, Mohning MP, Redente EF, Janssen WJ. Selective and inducible targeting of CD11b+ mononuclear phagocytes in the murine lung with hCD68-rtTA transgenic systems. Am J Physiol Lung Cell Mol Physiol (2016) 311:L87-100. doi:10.1152/ajplung.00141.2016
30. Jakubzick C, Gautier EL, Gibbings SL, Sojka DK, Schlitzer A, Johnson TE, et al. Minimal differentiation of classical monocytes as they survey steady-state tissues and transport antigen to lymph nodes. Immunity (2013) 39:599-610. doi:10.1016/j.immuni.2013.08.007

31. Huang QQ, Perlman H, Huang Z, Birkett R, Kan L, Agrawal H, et al. FLIP: a novel regulator of macrophage differentiation and granulocyte homeostasis. Blood (2010) 116:4968-77. doi:10.1182/blood-2009-11-252841

32. Gordy C, Pua H, Sempowski GD, He YW. Regulation of steady-state neutrophil homeostasis by macrophages. Blood (2011) 117:618-29. doi:10.1182/ blood-2010-01-265959

33. Casanova-Acebes M, Pitaval C, Weiss LA, Nombela-Arrieta C, Chevre R, A-González N, et al. Rhythmic modulation of the hematopoietic niche through neutrophil clearance. Cell (2013) 153:1025-35. doi:10.1016/j. cell.2013.04.040

34. Gibbings SL, Goyal R, Desch AN, Leach SM, Prabagar M, Atif SM, et al. Transcriptome analysis highlights the conserved difference between embryonic and postnatal-derived alveolar macrophages. Blood (2015) 126:1357-66. doi:10.1182/blood-2015-01-624809

35. Hochedlinger K, Yamada Y, Beard C, Jaenisch R. Ectopic expression of Oct- 4 blocks progenitor-cell differentiation and causes dysplasia in epithelial tissues. Cell (2005) 121:465-77. doi:10.1016/j.cell.2005.02.018

36. Zambrowicz BP, Imamoto A, Fiering S, Herzenberg LA, Kerr WG, Soriano P. Disruption of overlapping transcripts in the ROSA beta geo 26 gene trap strain leads to widespread expression of beta-galactosidase in mouse embryos and hematopoietic cells. Proc Natl Acad Sci U S A (1997) 94:3789-94. doi:10.1073/pnas.94.8.3789

37. Burnett SH, Beus BJ, Avdiushko R, Qualls J, Kaplan AM, Cohen DA. Development of peritoneal adhesions in macrophage depleted mice. J Surg Res (2006) 131:296-301. doi:10.1016/j.jss.2005.08.026

38. Bakri Y, Sarrazin S, Mayer UP, Tillmanns S, Nerlov C, Boned A, et al. Balance of MafB and PU.1 specifies alternative macrophage or dendritic cell fate. Blood (2005) 105:2707-16. doi:10.1182/blood-2004-04-1448

39. Wu X, Briseno CG, Durai V, Albring JC, Haldar M, Bagadia P, et al. Mafb lineage tracing to distinguish macrophages from other immune lineages reveals dual identity of Langerhans cells. J Exp Med (2016) 213:2553-65. doi:10.1084/jem.20160600

40. Gautier EL, Shay T, Miller J, Greter M, Jakubzick C, Ivanov S, et al. Gene-expression profiles and transcriptional regulatory pathways that underlie the identity and diversity of mouse tissue macrophages. Nat Immunol (2012) 13:1118-28. doi:10.1038/ni.2419

Conflict of Interest Statement: The authors declare that the research was conducted in the absence of any commercial or financial relationships that could be construed as a potential conflict of interest.

Copyright (C) 2017 McCubbrey, Allison, Lee-Sherick, Jakubzick and Janssen. This is an open-access article distributed under the terms of the Creative Commons Attribution License (CC BY). The use, distribution or reproduction in other forums is permitted, provided the original author(s) or licensor are credited and that the original publication in this journal is cited, in accordance with accepted academic practice. No use, distribution or reproduction is permitted which does not comply with these terms. 